\title{
Improvement of Stiffness Models for Laminated Rubber Bearing using Hyperelastic Finite Element Analysis
}

\author{
Rong-Can Hong ${ }^{\text {a, }}$, Kuo-Shen Chen ${ }^{\mathrm{b}}$ \\ ${ }^{\text {a }}$ Graduate Student, Department of Mechanical Engineering, National Cheng Kung University, No.1, Dasyue Rd., East \\ Dist., Tainan City 701, Taiwan \\ ${ }^{\mathrm{b}}$ Professor, Department of Mechanical Engineering, National Cheng Kung University, No.1, Dasyue Rd., East Dist., \\ Tainan City 701, Taiwan
}

*Corresponding Author: rchongme@gmail.com

\begin{abstract}
A detailed knowledge of rubber bearing behavior is essential for developing applications ranged from seismic isolation in civil engineering to the design of ultraprecision motion system in mechatronics. However, past rubber bearing models as essentially analytical which neglect many important details. Thus, a more sophisticated constitutive model should be developed. In this present study, a generic hyperelastic finite element model based on the Arruda-Boyce material constitutive equation is established for evaluating the loading capability of laminated rubber bearings. Simulations are performed to investigate the effective compression modulus and apparent shear modulus of rubber bearings with various shape factors. The numerical results are then compared with analytical solutions. The fundamental assumptions adapted by analytical models of rubber bearing are also examined. It is shown that the results of both finite element and linear analytical models agree to each other well for small compression. However, under large deformation, their results become to deviate from each other. Therefore, a correction function is added to existing analytical models. The analysis results presented in this work could provide more accurate prediction in designing the state-of-the-arts rubber bearing for related applications in precision engineering.
\end{abstract}

Keywords: Rubber bearing, compression modulus, apparent shear, finite element method, hyperelasticity.

\section{Introduction}

Laminated rubber bearings consist of rubber layers bonded by rigid steel shims. When the rubber layer is bonded between two steel plates, the steel plates restrict the lateral displacement of the rubber and result in a higher compression stiffness than the rubber without bonding. Thus, laminated rubber bearings can provide high vertical rigidity and still provide the same horizontal flexibility as elastomers. Due to these properties, they are widely used in earthquake engineering for seismic isolation ${ }^{(1)}$. Furthermore, due to their high anisotropic stiffness, rubber bearings are also increasingly applied in precision systems as an alternative to compliant mechanisms ${ }^{(2-4)}$. A typical application in precision engineering is shown in Fig. $1^{(4)}$. Four rubber pads were used to provide shear compliance in vertical motion while maintaining sufficient stiffness in planar direction due to compression. Compared to compliant mechanisms, laminated rubber bearings have a more compact form and a lower $\operatorname{cost}^{(3)}$. However, to predict the performance of laminated rubber bearings, their stiffness properties must be accurately known.

Many analytical solutions for predicting the stiffness of rubber bearings have been developed in recent decades. Two kinematics assumptions are usually adopted in such analytical models ${ }^{(5)}$ : first, planes parallel to the bonding plates before deformation remain planar after loading; second, lines normal to the bonding plates before deformation become parabolic after loading. Consequently, the stress distribution inside the rubber is usually assumed as a hydrostatic state. Based on those assumptions, Gent and Lindley $^{(5)}$ derived the compression stiffness of 
incompressible rubber bearings with infinite-strip shapes and circular shapes. Gent and Meinecke ${ }^{(6)}$ further extended the same method to analyze incompressible rubber bearings with square and other shapes. In particular, the stiffness anisotropy, or the the compression $\left(K_{C}\right)$ to shear stiffness $\left(K_{S}\right)$ ratio, denoted as $R_{C S}$ herein, for an N-layered rubber-metal laminate strip may be expressed as

$$
R_{C S} \equiv \frac{K_{C}}{K_{S}}=\frac{4 E_{0}\left(1+k N^{2} S^{2}\right)}{3 N G}
$$

where $E_{0}$ and $G$ are the initial Young's and shear modulus, respectively, $S$ the shape factor, and $k$ a material-dependent constant.

Eqn.(1) have been successfully served as the foundation of rubber bearing designs for decades. However, the assumption of incompressibility tends to overestimate the compression stiffness when the shape factor is high. Thus, Lindley ${ }^{(7)}$ applied an energy method to derive the compression stiffness for any Poisson's ratio. Tsai and Lee ${ }^{(8)}$ developed a pressure approach to derive the compression stiffness of rubber bearings with square shapes as

$$
\begin{aligned}
& \frac{E_{c}}{E}=1+\frac{4 v^{2}}{(1+v)(1-2 v)} \sum_{n=1}^{\infty} \frac{1}{\left[\left(\mathrm{n}-\frac{1}{2}\right) \pi\right]^{2}}\left\{\frac{\tanh \gamma_{n} a}{\gamma_{n} a}-\left[1-\frac{3(1-2 v)}{\gamma_{n}^{2} t^{2}+6(1-v)}\right] .\right. \\
& \left.\frac{\tanh \beta_{n} a}{\beta_{n} a}+\frac{\tanh \gamma_{n} b}{\overline{\gamma_{n}} b}-\left[1-\frac{3(1-2 v)}{-\gamma_{n}^{2} t^{2}+6(1-v)}\right] \frac{\tanh \overline{\beta_{n}} b}{\overline{\beta_{n}} b}\right\}
\end{aligned}
$$

where

$$
\begin{gathered}
\gamma_{n}=\left(\mathrm{n}-\frac{1}{2}\right) \frac{\pi}{b} \\
\bar{\gamma}_{n}=\left(\mathrm{n}-\frac{1}{2}\right) \frac{\pi}{a} \\
\beta_{n}=\sqrt{\gamma_{n}^{2}+\alpha^{2}} \\
\bar{\beta}_{n}=\sqrt{\bar{\gamma}_{n}^{2}+\alpha^{2}} \\
\alpha=\frac{1}{t} \sqrt{\frac{6(1-2 v)}{1-v}}
\end{gathered}
$$

In Eqns.(2-7) E and $v$ are elastic modulus and Poisson's ratio of rubber, respectively; $\mathrm{a}$ and $\mathrm{b}$ the half-length and half-width of cross-section, respectively. The solutions of Eqn.(2) were shown to be fairly accurate for materials with any Poisson ratios.

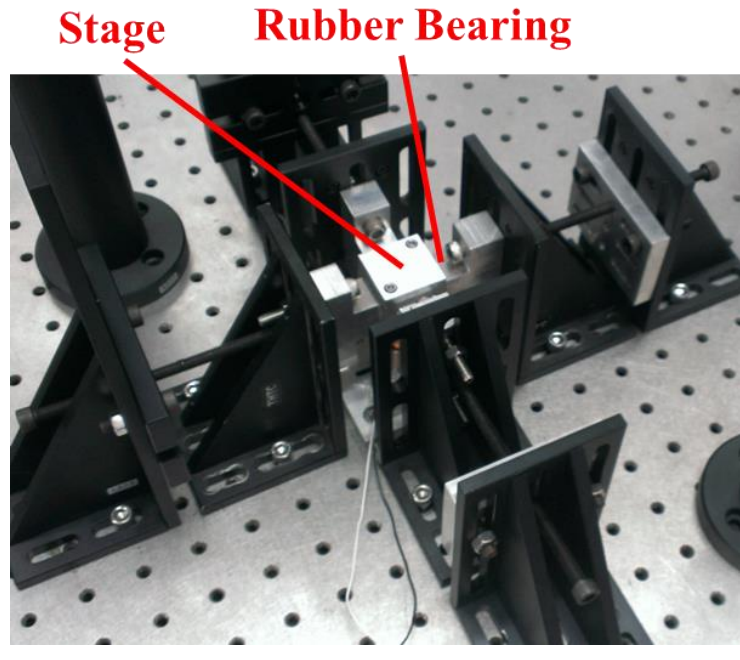

(a) A picture of the system

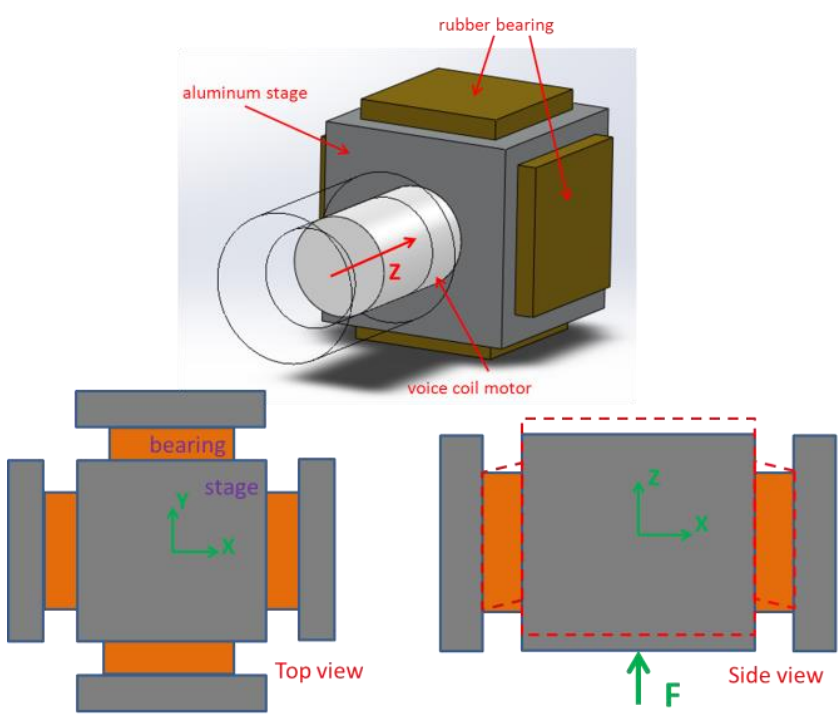

(b) Schematic plot of the rubber bearing stage

Fig. 1. A precision motion stage incorporated with rubber bearing design ${ }^{(4)}$.

However, the above mentioned kinematic assumptions are actually questionable. In Section 2, these kinematic assumptions will be examined by using finite element simulation. In addition, the development of these analytical models (both Lindley's and Tsai's) is based on elastic material i.e. rubber layer is assumed to be linear elastic. But when rubber bearing is under large deformation, the behavior is deviated from linear elasticity. Thus, the hyperelastic properties of rubbers are non-negligible as the load exceeds limit of small deformation. The difference between linear and hyperelastic material behavior will also be examined in Section 2.

In summary, the modeling errors resulted by possible inadequate kinematic assumptions and material constitutive laws may not be serious for traditional rubber bearing 
design used in civil engineering. However, for modern engineering applications, especially for applications in precision engineering, the scope of deformation and demand on accuracy is usually away from the applicable range of elastic model. Therefore, the analytical solutions proposed in (5-8) may not be suitable for precision engineering uses. Consequently, the analytical solutions need to be modified for practical engineering applications. However, it is too tedious and may not have a beautiful close form once all the effects are considered, as a result, it is desired to develop a modified model to cover board range of material nonlinearity while keeps the convenience of analytical solutions by in conjunction of numerical analysis. That is, in this work, a semi-empirical solution will be developed by adapting the core analytical formulation from Tsai ${ }^{(8)}$ with correction factors obtained by using finite element simulations.

This paper is aimed at developing more accurate predictions in design of rubber bearings for related applications in precision engineering. An improved stiffness model is developed to provide better parameters of controlling precision systems and reliability of structures. Accordingly, in the present study an improved analytical model are developed to enhance accuracy of prediction. Finite element simulations are performed and the results are used to improve analytical models. In this paper, a finite element model is developed for analysis of compression and shear stiffness of rubber bearing. The rubber layer is treated as Arruda-Boyce material ${ }^{(9)}$, which has been well-recognized as the most sophisticated hyperelastic models. The simulations will investigate the behavior of rubber bearing under compression or shear loading. And the compression modulus and apparent shear modulus will be obtained by the result of finite element analysis. The modified models are developed with combination of FEM and analytical solutions. The enhancement for accuracy of improved model is also investigated. The model developed in this paper can provide more applicable standard for design of rubber bearing.

The contribution of this study is therefore on providing a more reliable approach to design rubber bearings for particular purpose, especially mechanical engineering. This research offers a novel methodology of finding more comprehensive solutions while holds the convenience of analytical expressions for providing higher accuracy and short time in rubber bearing design.

\section{Problem Description and Development of Simulation Model}

In this paper, the model of rubber bearing is considered as a single rubber layer bonded by two steel shims, as is shown in Fig. 2. For precision engineering, rubber bearing with single layer is the most general and applicable model in this field. In this work, the geometry of model is constructed of rectangular rubber bearing, of which there are few analytical studies. The simulation is aimed to investigate properties of single-layered rubber bearing loaded by compression or shear force. The rubber layer is treated as hyperelastic material in this work. A general model is obtained from the simulation results to describe behaviors of rubber bearings under large deformation.

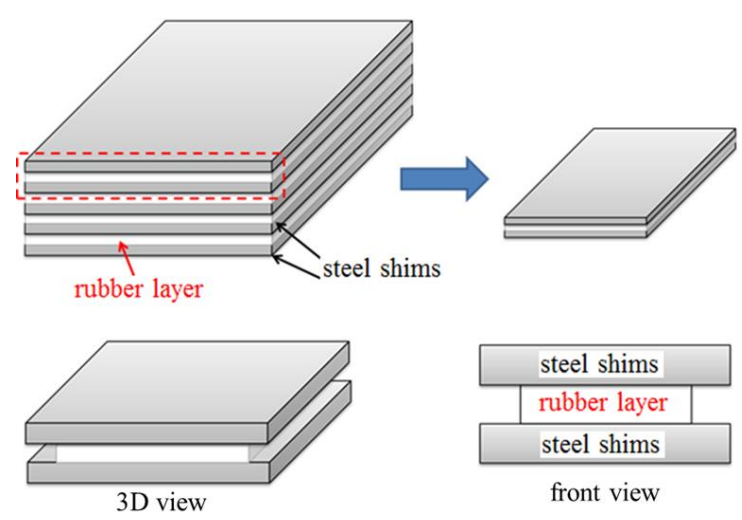

Fig. 2. Schematic plot of the model for rubber bearing.

Before conducting the model development, the key assumptions of developing classical rubber bearing equation should be examined. In addition, the influence of different material constitutive behavior should also be demonstrated.

Considering the finite element model for plane strain of a single layer rubber bearing subjected to compression loading, its deformation plots of the various rubbers at different stress levels are shown in Fig. 3. The bulge is initially a parabolic-shape, agreeing with Lindley's assumption. However, as the applied load increases, the deformation of rubber near the corners becomes substantial and the bulge gradually deviates from a parabolic shape. By checking the deformed mesh near the rubber edge, it is found that the assumption of the plane remains a plane is not satisfied. The situation becomes more severe for large deformation. Meanwhile, by examining the hydrostatic stress contour, it also reveals that the distribution is not uniform and this is also a key indicator of the failure of the kinematic assumption, as is shown in Fig. 4. 


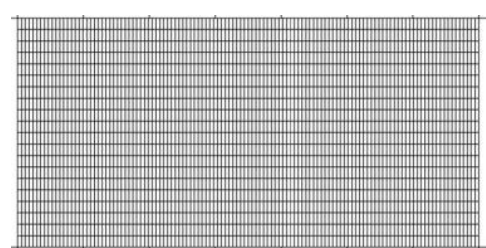

(a) undeformed mesh on the elastomer

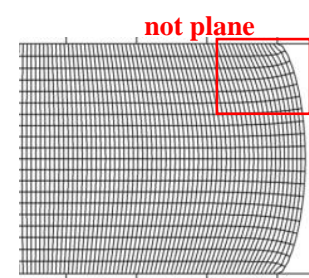

(b) deformed meshes at smaller compression

not plane

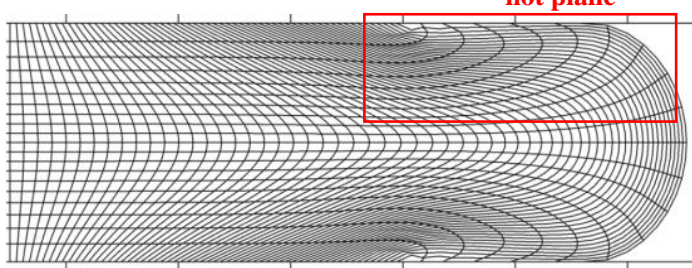

(c) deformed meshes at larger compression.

Fig. 3. Finite element deformation plot of an elastomer subjected to a compression force

Next, the different of material behavior is examined. A simple compression simulation is performed to study the behaviors of rubber bearings with the same initial modulus but different hyperelastic parameters. The shape factor is both set as 1 , but Arruda-Boyce parameter is set as $\mu=$ $0.33143 \mathrm{MPa}, \lambda_{\mathrm{m}}=7, D=0.012 \mathrm{Gpa}^{-1}$ and $\mu=0.28035 \mathrm{MPa}$, $\lambda_{\mathrm{m}}=2, D=0.012 \mathrm{Gpa}^{-1}$, respectively. In this case, the hyperelastic materials have the same initial elastic (1MPa) and shear modulus $(0.34 \mathrm{MPa})$ as those of the linear elastic material. The results of stress-strain curves are shown in Fig. 5. However, as one can see, for large deformation, the prediction from both models becomes non-negligible.

The above investigations clearly demonstrate the possible problems of the classical formation ${ }^{(5-7)}$ and the limitation of the modified expression ${ }^{(8)}$ and provide strong support for our proposed work.

In this study, three-dimensional finite element (FE) models are constructed of rectangular rubber bearings. The parameters are listed in Table 1. Each case has five different shape factors, namely $S=0.25,0.5,1.0,2.0,5.0$. The cross-sectional area is maintained as a constant $15^{*} 15 \mathrm{~mm}^{2}$ and the thickness is set as $t=15 ; 7.5,3.75$; $1.875 ; 0.75 \mathrm{~mm}$, respectively. The simulation models are established using the Arruda-Boyce model ${ }^{(9)}$ with parameter settings of $\mu=0.33143 \mathrm{MPa}, \lambda_{\mathrm{m}}=7, D=0.012 \mathrm{GPa}^{-1}$. The validity of the FE simulation results for the compression and shear modulus of rectangular bearings is verified by means of comparison with the analytical solutions of Tsai's ${ }^{(8)}$. The parameters of elastic material used in analytical solutions are set as: $E=1 \mathrm{MPa}$ and Poisson's ratio $v=0.49$, for the consistency of Arruda-Boyce model.
Abaqus are used to perform the finite element analysis of rubber bearing. The finite element model is shown in Fig. 6. The simulation model is meshed using 3D elements of second order (C3D20). The convergence analysis is based on compression load on rectangular rubber bearing under compression strain of $2 \%$ and with shape factor $S=1$, as is shown in Fig. 7. The rubber layers contain $20 * 20$ elements in the cross-section and 20 elements in the vertical direction. The parameters for finite element analysis are listed in Table 1.

\section{Analysis Approach}

Compression and shear stiffness of laminated rubber bearing are determined by effective compression modulus and apparent shear modulus defined as:

$$
\begin{gathered}
E_{C}=\frac{\sigma_{C}}{\varepsilon_{c}} \\
G_{a}=\frac{\tau_{a}}{\gamma_{a}}
\end{gathered}
$$

where $E_{\mathrm{C}}$ is the effective compression modulus, $\sigma_{\mathrm{C}}$ and $\varepsilon_{\mathrm{C}}$ is the effective compression stress and strain, respectively, $G_{a}$ the apparent shear modulus, and finally $\tau_{a}$ and $\gamma_{a}$ is the apparent shear stress and strain. From present analytical solutions ${ }^{(5-8)}$, these moduli are treated as constants in stress-strain curve, i.e. the stiffness of rubber bearing is independent of strain. However, results from FE simulations show that effective compression modulus and apparent shear modulus vary with strain. To find relationships between moduli and strain, stress-strain curves are obtained by simulations first. And then the curves of moduli to strain can be derived with stress-strain curves by:

$$
\begin{aligned}
& E_{c}\left(\varepsilon_{i}\right)=\frac{d \sigma_{C}\left(\varepsilon_{i}\right)}{d \varepsilon_{C}}=\frac{\Delta \sigma_{C}\left(\varepsilon_{i}\right)}{\Delta \varepsilon_{C}}=\frac{\sigma_{C}\left(\varepsilon_{i}\right)-\sigma_{C}\left(\varepsilon_{i-1}\right)}{\varepsilon_{C i}-\varepsilon_{C i-1}} \\
& G_{a}\left(\varepsilon_{i}\right)=\frac{d \tau_{a}\left(\gamma_{a i}\right)}{d \gamma_{a i}}=\frac{\Delta \tau_{a}\left(\gamma_{a i}\right)}{\Delta \gamma_{a i}}=\frac{\tau_{a}\left(\gamma_{a i}\right)-\tau_{a}\left(\gamma_{a(i-1)}\right)}{\gamma_{a i}-\gamma_{a(i-1)}}
\end{aligned}
$$

The simulation results will be compared with the solutions obtained by Tsai's model ${ }^{(8)}$. Eventually the general properties under high strain will be described. The relationship between moduli and strain is also obtained by curve fitting. The analytical models are then improved by adding functions of strain to develop a semi-empirical solution. The enhancement for accuracy of improved model is also discussed in subsequent sections. 


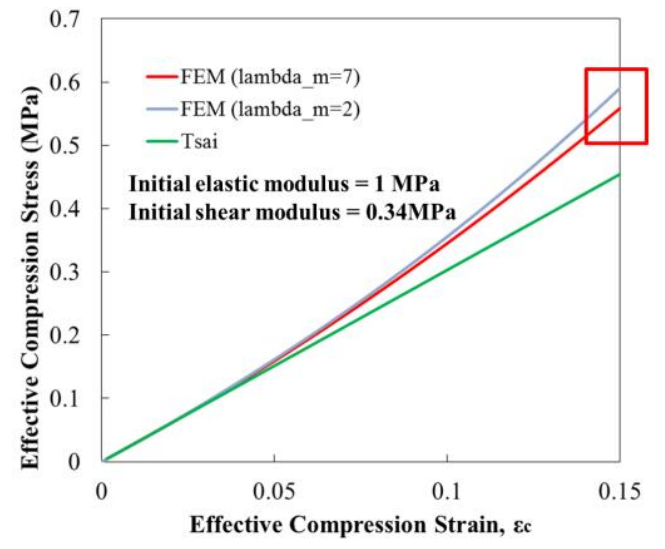

Fig. 5. Stress-strain curves of rubber bearings with the same initial modulus but different hyperelastic parameters.

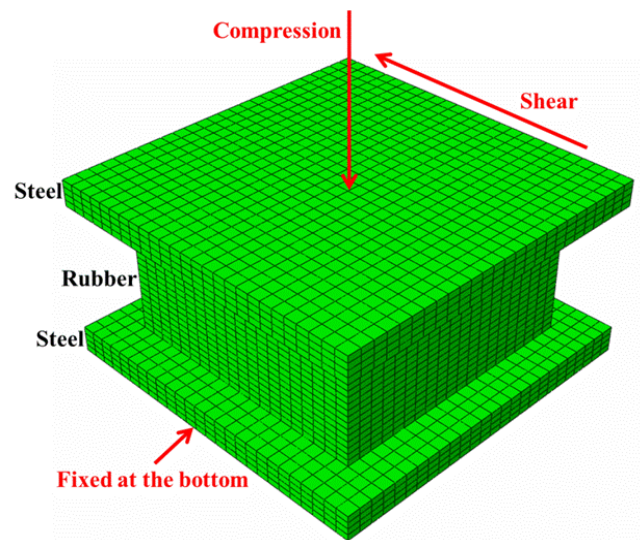

(a) 3D finite element model

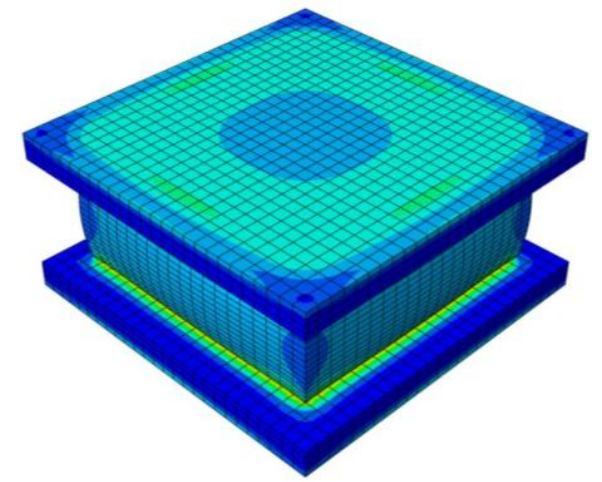

(b) Typical compression deformation stress contours

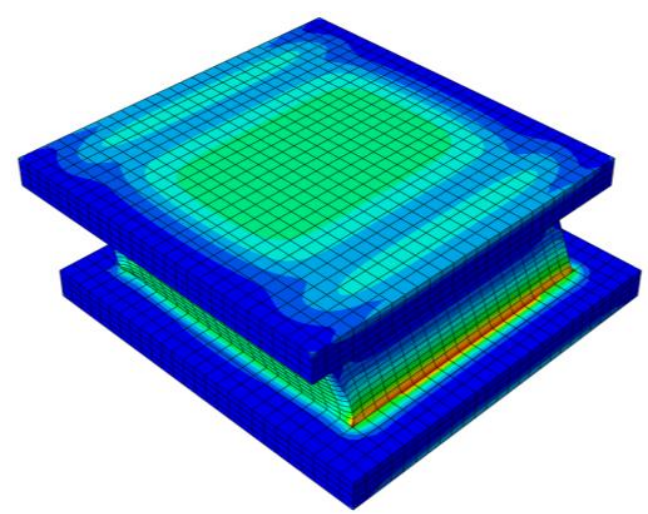

(c) Typical shear deformation stress contours

Fig. 6. Finite element model of rectangular rubber bearing.

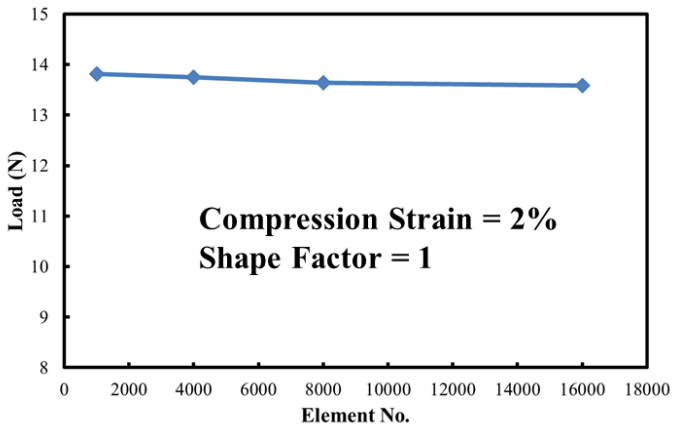

Fig. 7. Convergence analysis of the finite element model.

Table 1. Essential parameters for finite element model.

\begin{tabular}{|c|c|c|c|}
\hline \multicolumn{2}{|c|}{ Geometry } & \multicolumn{2}{|l|}{ Mesh } \\
\hline $\begin{array}{c}\text { Length of } \\
\text { cross-section } \\
(\mathrm{mm})\end{array}$ & 15 & Element Type & $\mathrm{C} 3 \mathrm{D} 20$ \\
\hline $\begin{array}{l}\text { Shape } \\
\text { factor, } S\end{array}$ & $\begin{array}{l}0.25 ; 0.5 \\
1 ; 2 ; 5\end{array}$ & $\begin{array}{l}\text { No. of elements } \\
\text { in rubber layer }\end{array}$ & 8000 \\
\hline $\begin{array}{l}\text { Thickness } \\
\text { (mm) }\end{array}$ & $\begin{array}{c}15 ; 7.5 ; \\
3.75 ; \\
1.875 ; 0.75 \\
\end{array}$ & $\begin{array}{l}\text { No. of elements } \\
\text { in steel layers }\end{array}$ & 5408 \\
\hline \multicolumn{4}{|c|}{ Hyperelastic Material Properties } \\
\hline \multicolumn{4}{|c|}{$\mu=0.33143 \mathrm{MPa}, \quad \lambda_{m}=7, \quad D=0.012 \mathrm{GPa}^{-1}$} \\
\hline \multicolumn{4}{|c|}{ Material of Steel } \\
\hline $\begin{array}{l}\text { Elastic } \\
\text { Modulus } \\
(\mathrm{GPa}) \\
\end{array}$ & 200 & Poisson's Ratio & 0.3 \\
\hline
\end{tabular}

\section{Simulation with different types of loads}

\subsection{Compression Analysis}

The strain-stress curves obtained from FE analysis and Tsai's solution for rectangular rubber bearing with applied compression load are shown in Fig. 8. As is shown in Fig. 8, the FE results and Tsai's solutions are consistent under low $\varepsilon_{\mathrm{C}}$ (about $2-5 \%$ ). However, the results of FE and Tsai's solutions are deviated as compression strain increases. Therefore, the nonlinear behaviors of rubber bearings are non-negligible under high deformation. From the analysis, the actual loads are underestimated in comparison of analytical solutions. To investigate the nonlinearity of stiffness, Eqn. (10) is used to produce curves of moduli to strain, as is Fig. 9 shown. From Fig. 9, the effective compression modulus is getting higher as the compression strain increases. So it is confirmed that effective compression modulus is sensitive to compression strain. Thus, the magnitude of compression strain should be considered to obtain more accurate stiffness of rubber bearing. Furthermore, a semi-empirical equation will be developed in section 5 . 


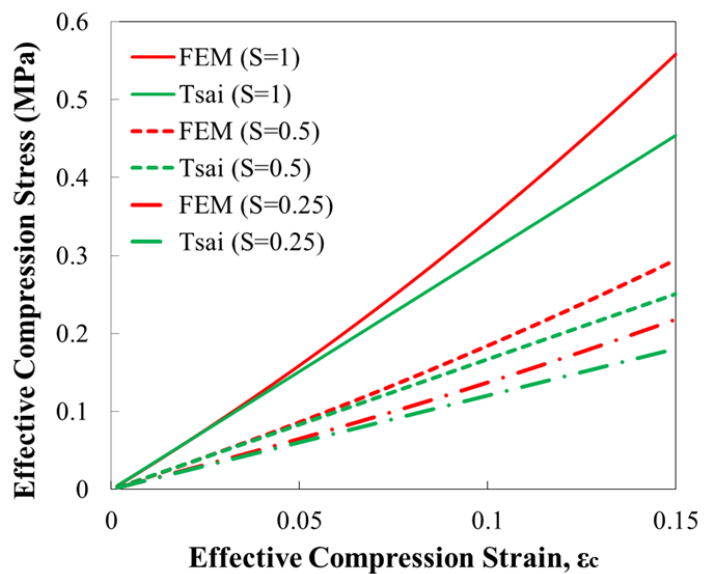

Fig. 8. Stress-strain curves of compression analysis for rectangular rubber bearings.

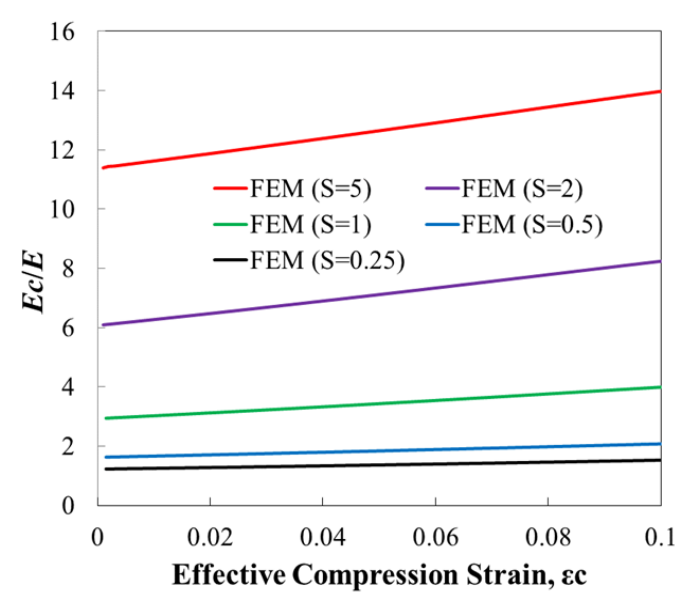

Fig. 9. Curves of effective compression modulus to compression strain.

\subsection{Shear Analysis}

The strain-stress curves obtained from FE analysis for rectangular rubber bearing with applied shear force are shown in Fig. 10. In present analytical studies, apparent shear modulus is treated as the same as shear modulus of rubber material used in rubber bearing. However, from simulation results in Fig. 10, the load curves are different for low shape factors. Because rubber bearings of low shape factors are widely used in mechanical engineering, the assumption of apparent shear modulus in analytical studies is not suitable. In section 5, a semi-empirical equation of relationship between apparent shear modulus and shape factor is developed for applications in mechanical engineering. To investigate sensitivity of shear stiffness to apparent shear strain, Eqn. (11) is used to obtain moduli which differ with strain. And then fluctuation of apparent shear modulus with strain is defined as:

$$
\text { Fluctuation }=\frac{\left(\frac{G_{a}}{G}\right)-\left(\frac{G_{a}}{G}\right)_{\gamma_{a}=10 \%}}{\left(\frac{G_{a}}{G}\right)_{\gamma_{a}=10 \%}}
$$

The analysis of fluctuation is shown in Fig. 11. From the analysis results, the fluctuation is within $1 \%$, so it is confirmed that apparent shear modulus is not sensitive to apparent shear strain. Therefore, shear stiffness can be assumed to be independent of shear strain.

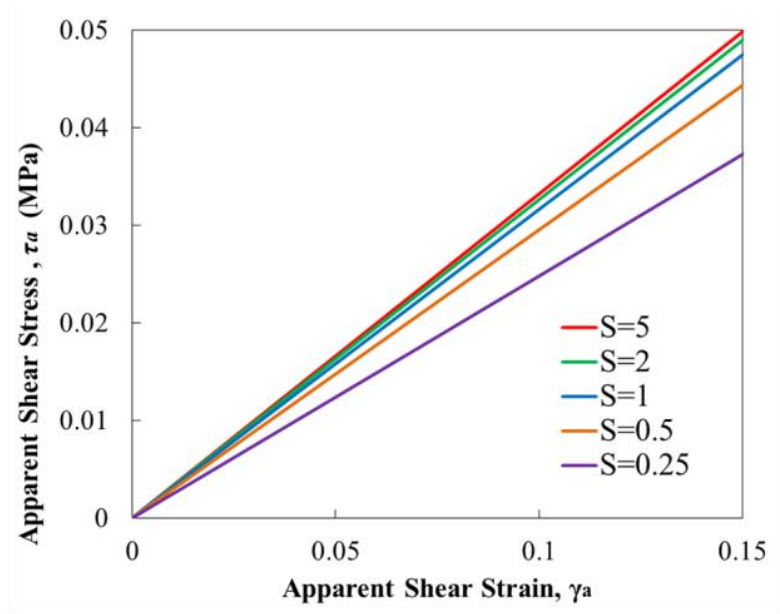

Fig. 10. Stress-strain curves of shear analysis for rectangular rubber bearings.

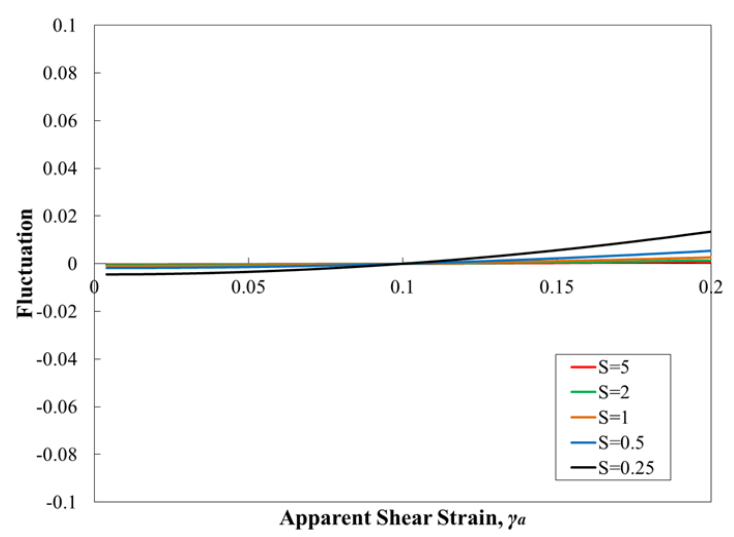

Fig. 11. Fluctuation of apparent shear modulus to shear strain.

\section{Improvement of Analytical Models}

\subsection{Semi-empirical Equation of effective compression modulus}

From the analysis in Section 4.1, effective compression modulus of rubber bearing is related to compression strain. By curve fitting, effective compression modulus is a quadratic function of compression strain, as is shown in Fig. 
12. In this work, a semi-empirical solution is developed on Tsai's analytical solution with a correction function of strain:

$$
\left(E_{C}\right)_{\text {modified }}=\left(E_{C}\right)_{T s a i}\left[a \varepsilon_{C}^{2}+b \varepsilon_{C}+c\right]
$$

where $\left(E_{\mathrm{C}}\right)_{\text {modified }}$ is the improved solution of effective compression modulus, $\left(E_{\mathrm{C}}\right)_{T s a i}$ is the solution of Tsai's analytical model, $\varepsilon_{\mathrm{C}}$ are effective compression strain and $a$, $b, c$ are coefficients related to material properties of rubber and geometry of rubber bearings. The comparison between FE analysis results, improved semi-empirical model and original analytical solution of Tsai's is shown in Fig. 13. From the results of improvement, the modified model of compression modulus can provide better performance in accuracy.

\subsection{Semi-empirical Equation of apparent shear modulus}

From the analysis in Section 4.2, apparent shear modulus of rubber bearing is a function of shape factor. Based on the results of Section 4.2, an empirical equation of apparent shear modulus is developed:

$$
G_{a}=G\left(1-\frac{A}{S}\right)
$$

where $G_{a}$ and $G$ are the real and apparent shear moduli, $S$ the shape factor, A the empirical coefficient based on FE analysis results. For example, $A=0.065$ obtained from the analysis in Section 4.2, as shown in Fig.14. Eqn. (13) can be used for design of precision system with rubber bearing to provide better accuracy.

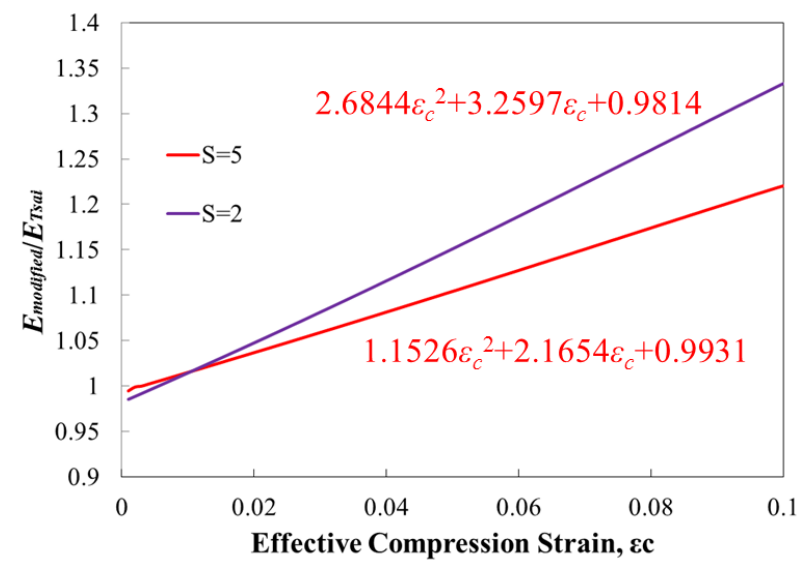

Fig. 12. Curve fitting of effective compression modulus to compression strain.

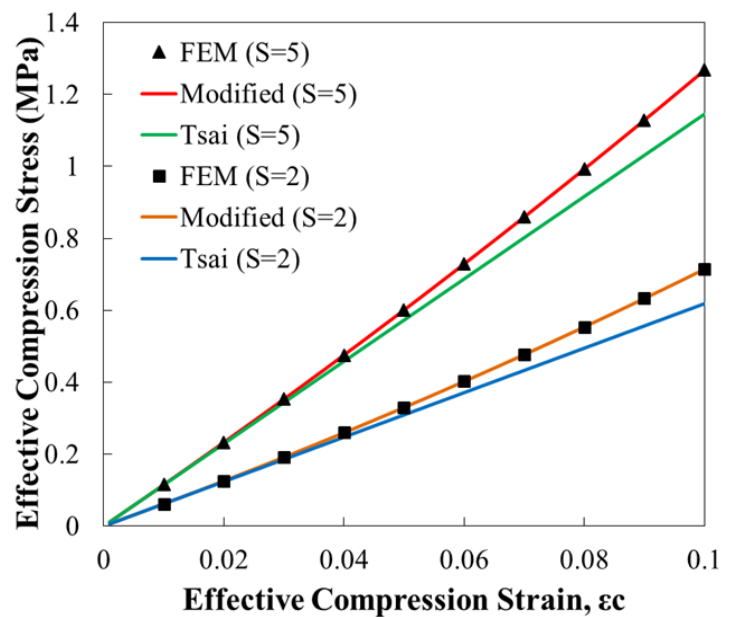

Fig. 13. Comparison of improved model and Tsai's analytical solution.

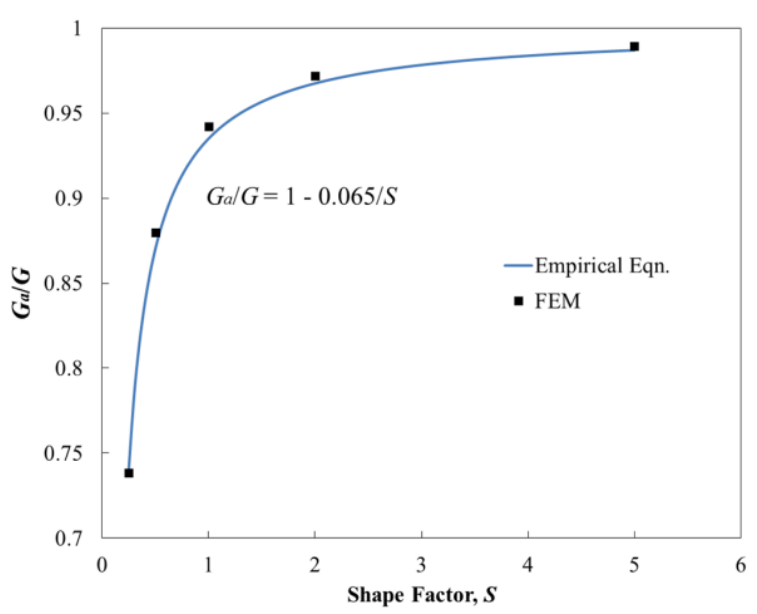

Fig. 14. Comparison of empirical equation and finite element analysis for shear analysis.

\section{Discussion}

The suitability of traditional kinematic assumptions in rubber bearing analysis is firstly examined and the effect of material behavior is followed. Both of the investigations shows the possible defects of the foundation of previous rubber bearing stiffness formulations.

In our investigation, as shown in Section 4, different hyperelastic materials have quite different behavior in high strain. That is, the elastic model is only applicable in the range of small deformation, even the initial modulus is the same as the hyperelastic material. Furthermore, the analysis in Section 4.1 shows that effective compression stiffness increase as compression strain getting higher, i.e. compression load in high strain is larger than prediction of analytical model. Therefore, the study in Section 5.1 develops a modified solution of compression modulus 
based on present analytical model and results of FE simulation. The improved model covers the nonlinearity of modulus with strain while keeps the convenience of analytical solutions. This modified solution can be introduced directly into control systems in ultraprecision engineering to obtain better accuracy and reliability.

For shear analysis, apparent shear modulus is assumed as the same as shear modulus of rubber material used in rubber bearing in present analytical studies. The results in Section 4.2 show that this assumption is not suitable for low shape factors, which are commonly chosen for rubber bearings in mechanical engineering. Thus, Section 5.2 gives an empirical equation for apparent shear modulus and also shows good accuracy of the equation compared with FE model in Fig. 14.

However, it should also emphasis here that there in order to achieve a comprehensive modification model, more numerical analyses should be conducted. Here only the effect of shape factor is considered. In the future, the effect of initial shear modulus should also be investigated. Nevertheless, the procedure is the same. Thus the contribution of this study is therefore providing a flow for more accurate prediction of stiffness of rubber bearings. This paper discusses the hyperelasic behavior of rubber bearing in large deformation with shape factors and hyperelastic parameters, respectively. The applicable range of present analytical solutions is extended with the FE analysis to enhance the accuracy performance of applications in precision systems. This work gives a general methodology for design of laminated rubber bearings, its applications with certain rubber material and more complicated loading types can be studied in future work. The viscoelastic effect of rubber bearings combined with hyperelastic models is also to be considered in next stage.

\section{Conclusion}

The behaviors of laminated rubber bearings to compression and shear are critical for modern rubber bearing applications. Using hyperelastic finite element analysis, this work examines the behavior of single-layered rubber bearing under large deformation, finding that the present analytical solution for describing rubber bearing behavior may not be adequate due to inappropriate assumptions in developing the formulas. Consequently, it is found that the compression stiffness may deviate significantly from the analytical solution at typical operating strain levels. Also, it is found that apparent shear modulus differs for various shape factors, especially low shape factors. Based on the FE analysis, semi-empirical equations for effective compression and apparent shear moduli are developed for better performance in prediction of stiffness of rubber bearings. The improved model presented in this work could provide more accurate prediction in designing the state-of-the-arts rubber bearing for related applications in precision engineering.

\section{Acknowledgment}

This research was partially supported by the Ministry of Science and Technology in Taiwan through Grant NSC 101-2221-E-006-032-MY3.

\section{References}

(1) J. M. Kelly, Mechanics of Rubber Bearings for Seismic and Vibration Isolation, $1^{\text {st }}$ ed. John Wiley \& Sons Ltd, 2011.

(2) A. E. Barton and D. L. Trumper, "Study of rubber bearings and its applicability in precision machines," Proc. 2005 ASPE Annual Meeting, Norfolk, VA, October 14, 2005

(3) A. E. Barton-Martinelli, Rubber Bearings for Precision Positioning Systems, Master Thesis, Department of Mechanical Engineering, Massachusetts Institute of Technology, USA, 2005

(4) Y.-C. Teng, Analysis, Design and Control of a Novel Elastomeric Bearing Positioning Stage, Master Thesis, Department of Mechanical Engineering, National Cheng Kung University, Taiwan, 2015

(5) A. N. Gent and P. B. Lindley, "The compression of bonded rubber blocks," Proc. Instn. Mech. Engrs, Vol. 173, pp. 111-122, 1959.

(6) A. N. Gent and E. A. Meinecke, "Compression, bending, and shear of bonded rubber blocks," Polymer Engineering \& Science, vol. 10, pp. 48-53, 1970.

(7) P. B. Lindley, "Compression module for blocks of soft elastic material bonded to rigid end plates," Journal of Strain Analysis, vol. 14, pp. 11-16, 1979.

(8) H.-C. Tsai, "Compression analysis of rectangular elastic layers bonded between rigid plates," International Journal of Solids and Structures ,vol. 42, pp.3395-3410, 2005.

(9) Abaqus theory manual, Version 6.10, Stimula, 2009. 